\title{
Effect of contact statistics on electrical contact resistance
}

\author{
Yong Hoon Jang ${ }^{\text {a) }}$ \\ School of Mechanical Engineering, Yonsei University, Shinchon-Dong 134, Seodaemun-Gu, Seoul, Korea \\ J. R. Barber ${ }^{\text {b) }}$ \\ Department of Mechanical Engineering, University of Michigan, Ann Arbor, Michigan 48109-2125
}

(Received 4 April 2003; accepted 10 September 2003)

\begin{abstract}
The flow of electrical current through a microscopic actual contact spot between two conductors is influenced by the flow through adjacent contact spots. A smoothed version of this interaction effect is developed and used to predict the contact resistance when the statistical size and spatial distribution of contact spots is known. To illustrate the use of the method, an idealized fractal rough surface is defined using the random midpoint displacement algorithm, and the size distribution of contact spots is assumed to be given by the intersection of this surface with a constant height plane. With these assumptions, it is shown that including finer scale detail in the fractal surface, equivalent to reducing the sampling length in the measurement of the surface, causes the predicted resistance to approach the perfect contact limit. () 2003 American Institute of Physics.
\end{abstract}

[DOI: $10.1063 / 1.1622995]$

\section{INTRODUCTION}

When two large conductors make perfect electrical contact over a small circular area of radius $a$, there will be a constriction resistance to electrical flow between them of $\rho / 2 a$, where $\rho$ is the electrical resistivity. This equation is widely used in the design and study of electrical contacts. However, if the contacting bodies have rough surfaces, contact will rarely be restricted to a single area. Instead, there will be contact at a multitude of microscopic "actual" contacts clustered within a macroscopic "nominal" or "apparent" contact area. Greenwood ${ }^{1}$ has analyzed such clusters, treating a number of distributions of size and spacings, and has confirmed an earlier suggestion by Holm ${ }^{2}$ that the combined effect of the local constriction and the clustering is to generate a resistance

$$
R=\rho\left(\frac{1}{2 N a}+\frac{1}{2 \alpha}\right)
$$

where $N$ is the number of circular contact spots and $\alpha$ is the radius of the cluster.

Many authors have attempted to generalize Greenwood's results to define the electrical and thermal conductance in the presence of clusters of microcontacts. Boyer et al. ${ }^{3}$ developed a model based on the assumptions that the number of contact spots is small and that the total area of actual contact is close to the nominal contact area. Malucci ${ }^{4}$ simulated degradation of the electrical conductance of aging contacts by introducing an interfacial film whose thickness is a statistical function of asperity deformation. In a later study, ${ }^{5}$ he extended this approach by including the effects of contact force, microhardness, and geometry as constraints in calculating the density of contact spots. Bryant ${ }^{6}$ investigated a

\footnotetext{
a) Author to whom correspondence should be addressed; electronic mail: jyh@yonsei.ac.kr

${ }^{\mathrm{b})}$ Electronic mail: jbarber@engin.umich.edu
}

hypothetical corrosive fretting failure mechanism in electrical contacts, incorporating asperity deformation, surface topography, and other factors. Contact resistance has been computed numerically by Nakamura ${ }^{7}$ for a system of two cubic electrodes contacting through a set of square contact spots, while Boyer ${ }^{8}$ has extended the Greenwood formula to include the presence of interfacial films by considering the rectangular juxtaposition of square spots of equal size and square ring-shaped spots.

Equation (1) provides a good approximation to the electrical contact resistance for a deterministic distribution of contact spots of known size and location, but information about the distribution of asperities is most likely to be statistical in nature, since surface roughness is essentially a random process. Furthermore, surface roughness descriptions are typically multiscale in nature, and on a sufficiently fine scale the number of discrete contact spots is likely to be too large to permit an efficient deterministic calculation. In the present paper, we shall develop a statistical version of Greenwood's equation, in which the summation is replaced by an integral over the nominal contact area with a kernel that depends on the statistical properties of the distribution. We shall then test the predictions of the theory by comparison with a discrete deterministic realization developed using the random midpoint displacement algorithm. In particular, we shall investigate the effect on the predicted contact resistance of the sampling length on the model surface, using recent results due to $\mathrm{Jang}^{9}$ for relations between two- and threedimensional properties of random surfaces.

\section{STATISTICAL IMPLEMENTATION OF GREENWOOD'S EQUATION}

Greenwood's result is based on the approximation of the potential field due to current flow through a microscopic contact spot by that due to a point current source in all locations 


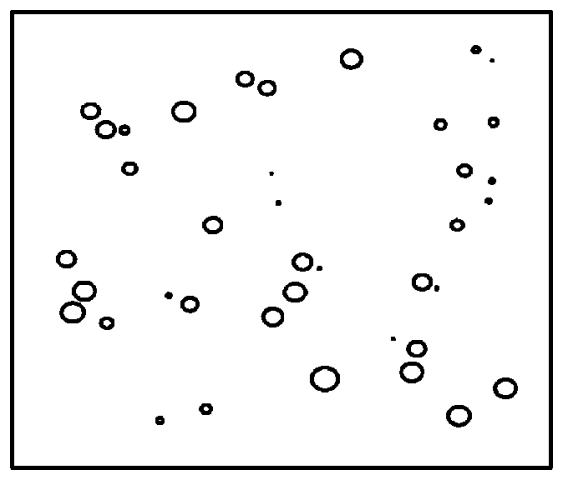

FIG. 1. Configuration of contact.

other than the immediate vicinity of the contact spot. Thus, the potential $\phi_{j}$ at the $j$ th contact spot in a set of $N$ randomly disposed contact spots as shown in Fig. 1 is

$$
\phi_{j}=\frac{\rho I_{j}}{4 a_{j}}+\frac{\rho}{2 \pi} \sum_{i \neq j} \frac{I_{i}}{s_{i j}},
$$

where $I_{i}$ is the current through the $i$ th contact spot, $a_{i}$ is its radius, $s_{i j}$ is the distance between the centers of the $i$ th and $j$ th contact spots, and the summation is performed over all the $N$ contact spots except $i=j$.

\section{A. The base potential} (2):

We will define the base potential $\bar{\phi}_{j}$ through the relation

$$
\bar{\phi}_{j} \equiv \phi_{j}-\frac{\rho I_{j}}{4 a_{j}}=\frac{\rho}{2 \pi} \sum_{i \neq j} \frac{I_{i}}{s_{i j}} .
$$

With this notation, we have

$$
I_{j}=\frac{4 a_{j}}{\rho}\left(\phi_{j}-\bar{\phi}_{j}\right)
$$

and, using this result to substitute for $I_{i}$ in Eq. (3), we obtain

$$
\bar{\phi}_{j}=\frac{2}{\pi} \sum_{i \neq j} \frac{a_{i}\left(\phi_{i}-\bar{\phi}_{i}\right)}{s_{i j}} .
$$

\section{B. Integral form of the equation}

Suppose that in some nominal area $A$ there exists a single circular contact spot and that the probability of its radius being between $a$ and $a+\delta a$ and of its center being located in the rectangle defined by the lines $x, x+\delta x, y, y$ $+\delta y$ is $h(x, y, a) \delta x \delta y \delta a$, where $h(x, y, a)$ is a probability distribution function that satisfies the equation

$$
\iint_{A}\left[\int_{0}^{\infty} h(x, y, a) d a\right] d x d y=1 \text {. }
$$

A similar definition can be used for the case where there are $n$ contact spots per unit nominal area, in which case the probability of a contact spot of radius $a, a+\delta a$ having its center within the infinitesimal rectangle will be $n A h(x, y, a) d a d x d y$. This definition implicitly assumes that the distribution is uncorrelated-i.e., that the probability of a contact spot at $(x, y)$ is unaffected by the actual occurrence of a contact spot at a nearby point. The consequences of this assumption will be discussed in Sec. VII.

The base potential at the point $x, y$ due to the distribution $h(x, y, a)$ can now be written

$$
\begin{aligned}
& \bar{\phi}(x, y) \\
& =\iint_{A} \int_{0}^{\infty} \frac{2 n A h(\xi, \eta, a)[\phi(\xi, \eta)-\bar{\phi}(\xi, \eta)] \operatorname{adad} \xi d \eta}{\pi \sqrt{(x-\xi)^{2}+(y-\eta)^{2}}},
\end{aligned}
$$

where the domain of integration is the nominal contact area and the range of contact spot radii.

If the integral with respect to $a$ can be performed, defining the function

$$
\bar{h}(\xi, \eta) \equiv \int_{0}^{\infty} n A h(\xi, \eta, a) a d a,
$$

then

$$
\bar{\phi}(x, y)=\iint_{A} \frac{2 \bar{h}(\xi, \eta)[\phi(\xi, \eta)-\bar{\phi}(\xi, \eta)] d \xi d \eta}{\pi \sqrt{(x-\xi)^{2}+(y-\eta)^{2}}} .
$$

\section{THE BOUNDARY VALUE PROBLEM}

If two half spaces make electrical contact at a number of areas on their common plane surface, the potential problems in the two bodies will be geometrically similar and the actual contact areas will form an equipotential surface. In particular, the potential difference between this surface and the extremity of body $i(i=1,2)$ will be

$$
\phi=\frac{U \rho_{i}}{\rho_{1}+\rho_{2}},
$$

where $\rho_{i}$ denotes the resistivity of the material of body $i$ and $U$ is the potential difference between the extremities of the two bodies. In more general problems, $\phi$ may not be constant. For example, if one of the bodies conducts a current in a direction tangential to the common interface, $\phi$ will be a linear function of $\xi, \eta$.

Thus, $\phi(\xi, \eta)$ is a known function, as is $h(\xi, \eta, a)$, and hence we can determine the function

$$
f(x, y) \equiv \iint_{A} \frac{2 \bar{h}(\xi, \eta) \phi(\xi, \eta) d \xi d \eta}{\pi \sqrt{(x-\xi)^{2}+(y-\eta)^{2}}} .
$$

It follows that the base potential $\bar{\phi}$ is the solution of

$$
\iint_{A} \frac{2 \bar{h}(\xi, \eta) \bar{\phi}(\xi, \eta) d \xi d \eta}{\pi \sqrt{(x-\xi)^{2}+(y-\eta)^{2}}}+\bar{\phi}(x, y)=f(x, y),
$$

which is a singular integral equation of the second kind, for which various solution methods are available.

Once $\bar{\phi}(x, y)$ has been determined from this equation, the current through each individual contact spot is defined in the discrete formulation of Eq. (4) so that the current through all contact spots can be summed as 


$$
I \equiv \sum_{j=1}^{N} I_{j}=\sum_{j=1}^{N} \frac{4 a_{j}}{\rho}\left(\phi_{j}-\bar{\phi}_{j}\right)
$$

The integral form of this equation can be written as

$$
\begin{aligned}
I= & \frac{4}{\rho} \iint_{A} \int_{0}^{\infty} n A h(\xi, \eta, a)[\phi(\xi, \eta) \\
& -\bar{\phi}(\xi, \eta)] a d a d \xi d \eta \\
= & \frac{4}{\rho} \iint_{A} \bar{h}(\xi, \eta)[\phi(\xi, \eta)-\bar{\phi}(\xi, \eta)] d \xi d \eta .
\end{aligned}
$$

We can also define the local mean current density as

$$
i(x, y)=\frac{4}{\rho} \bar{h}(x, y)[\phi(x, y)-\bar{\phi}(x, y)] .
$$

Notice that $i(x, y)$ is averaged over the local discontinuities associated with the actual contact areas, but it will vary over the nominal contact area. Statistically, it can also be regarded as the expected value of current density at the point $(x, y)$.

\section{INTERPRETATION OF THE FUNCTION $\bar{h}(\xi, \eta)$}

Suppose that the spatial distribution of contact spots and the size distribution are uncorrelated, so that $h(\xi, \eta, a)$ can be written in the normalized separated variable form

$$
h(\xi, \eta, a)=h_{1}(\xi, \eta) h_{2}(a),
$$

where

$$
\iint_{A} h_{1}(\xi, \eta) d \xi d \eta=1, \quad \int_{0}^{\infty} h_{2}(a) d a=1 .
$$

We then have

$$
\bar{h}(\xi, \eta)=n A h_{1}(\xi, \eta) \int_{0}^{\infty} h_{2}(a) a d a=n A h_{1}(\xi, \eta) \bar{a}
$$

from Eq. (12), where $\bar{a}$ is the mean value of $a$. If the distribution function $h_{1}(\xi, \eta)$ is uniform in $A$, we have

$$
h_{1}(\xi, \eta)=\frac{1}{A}
$$

from Eq. (18) and hence

$$
\bar{h}(\xi, \eta)=n \bar{a} .
$$

More generally, the function $\bar{h}(\xi, \eta)$ is equal to the product of the number of contact spots per unit area and the mean radius, both of which may be functions of position.

\section{MICROCONTACT SPOT DISTRIBUTION}

Equations (10), (12), and (15) permit us to determine the electrical contact resistance

$$
R_{e}=\frac{U}{I}
$$

for any rough surface contact problem, provided we can determine the corresponding statistical distribution functions $h_{1}(\xi, \eta)$ and $h_{2}(a)$. Various methods exist for this purpose. For example, we might use an asperity model theory such as those due to Greenwood and Williamson ${ }^{10}$ for flat surfaces or Greenwood and Tripp ${ }^{11}$ for nonconforming surfaces. In the present paper, we shall illustrate the method by making the assumption that the distribution of contact spots is defined by the set of "islands" generated by cutting through the rough surface at constant height. This assumption was used by Majumdar and Bhushan ${ }^{12}$ in their fractal theory of contact and is related to the concept of "bearing area," which is arguably appropriate when the contact deformations are predominantly plastic.

For this purpose, we generated a randomly rough surface using the random midpoint displacement algorithm (RMD) $\left(\right.$ Voss $\left.^{13}\right)$. Suppose the values of the process are defined at the nodal points of a square grid. The grid is now subdivided by introducing new nodal points at the midpoints. The value of the process at each midpoint is determined as the sum of the average of the two adjacent end points and a zero mean random process with a Gaussian distribution. This procedure of subdivision is applied recursively, and the standard deviation of the random process at each scale is chosen so as to ensure that the algorithm generates a self-affine fractal surface.

Starting with a square of dimension $L \times L, m$ applications of the algorithm will generate a square grid of $\left(2^{m}\right.$ $+1) \times\left(2^{m}+1\right)$, corresponding to a fractal surface measured with a sampling length of $L / 2^{m}$.

Figure 2(a) shows a typical rough surface generated by this algorithm in the unit square and Fig. 2(b) shows the corresponding bearing area ratio $B(z)$. The bearing area ratio is defined as the proportion of the surface above the height $z$. The $z$ axis in these figures is normalized with respect to the standard deviation $\sigma$ (i.e., the rms roughness) of the resulting surface.

Figure 2(c) shows the contact spots defined by cutting through the surface at the level where the bearing area ratio is $5 \%$ [i.e., $B(z)=0.05$ ], with a grid size (sampling length) of $1 / 2^{7}$. A total of 27 contact spots are identified, but they are clearly not circular, as required by the analysis of Sec. II. A distribution function for "equivalent" contact radii might be obtained by defining a set of circles whose areas are equal to those of the islands in Fig. 2(c). However, we note that in most cases the complete topographical description implied in Fig. 2(a) is not available. Instead, we typically have profilometer output, which is equivalent to a sampling of the surface along one or more lines. This permits the bearing area ratio [Fig. 2(b)] to be determined, but information about the distribution of islands $h_{2}(a)$ must be deduced from the corresponding distribution of line segments $f(l)$ above a given height in the profile. Jang ${ }^{9}$ has shown that a distribution $h_{2}(a)$ of circular contact spots will lead to a distribution $f(l)$ of line segments above the specified height, where

$$
h_{2}(a)=-\frac{2 \bar{a}}{\pi} \frac{d}{d a} \int_{a}^{\infty} \frac{f(l)}{\sqrt{l^{2}-a^{2}}} d l,
$$

and the mean radius of the circles is 

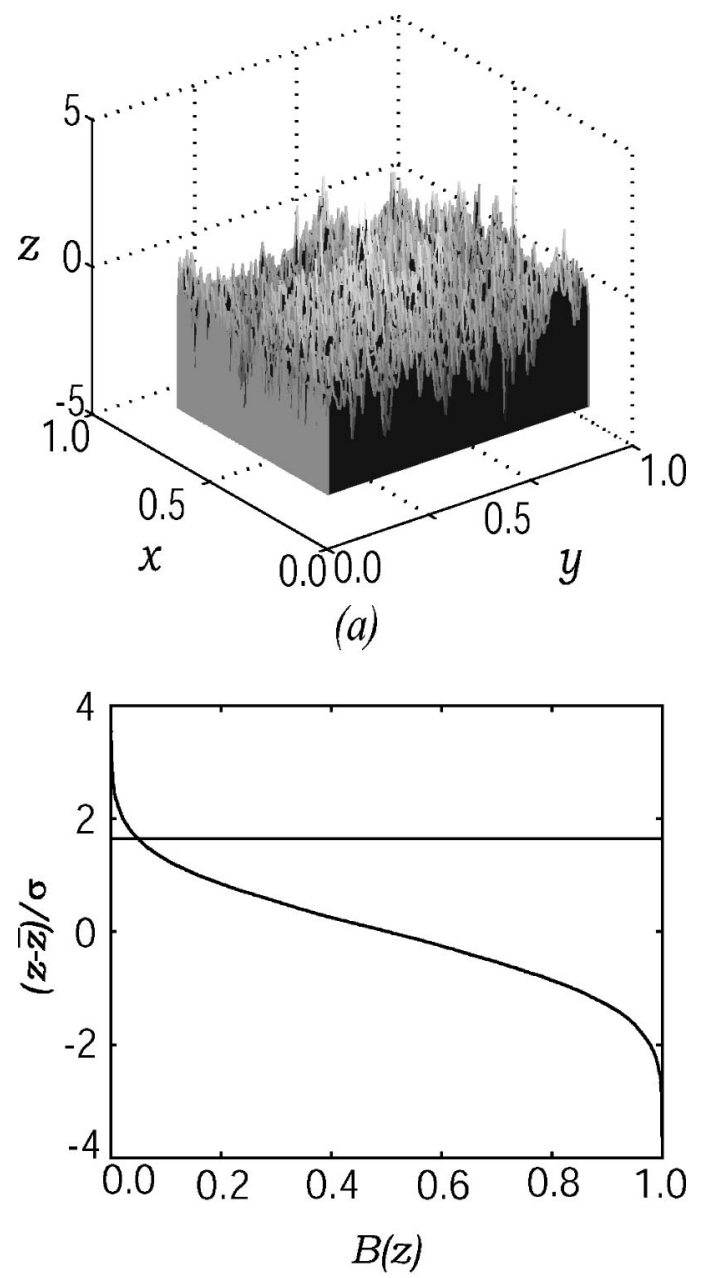

(b)

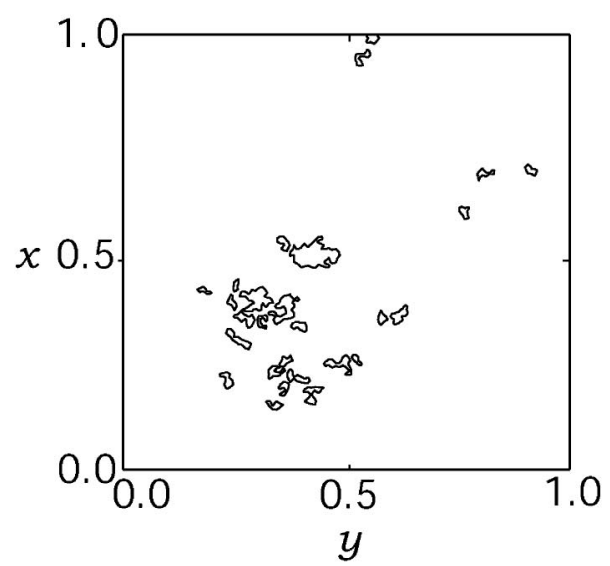

(c)

FIG. 2. Rough surface generated by the RMD algorithm. (a) Threedimensional view; (b) the corresponding bearing area ratio $B(z)$; (c) the set of islands generated by cutting through the surface at $B(z)=0.05$.

$$
\bar{a}=\pi /\left[2 \int_{0}^{\infty} \frac{f(l)}{l} d l\right] .
$$

The number of contact spots $n$ per unit area is

$$
n=\frac{2 n_{c}}{\bar{a}}
$$
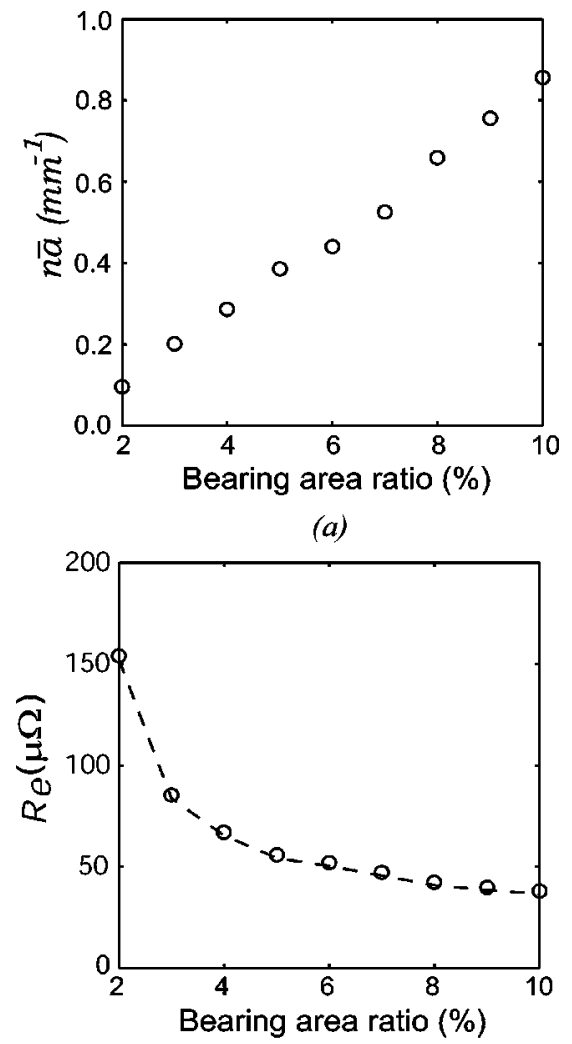

(b)

FIG. 3. Variation of (a) the function $\bar{h}=n \bar{a}$ from Eq. (21) and (b) the electrical contact resistance $R_{e}$ with bearing area ratio $B(z)$. The dashed line in (b) was obtained from Eq. (27).

where $n_{c}$ is the number of line segments per unit length. ${ }^{9}$

To utilize the above equations, we first sample the model surface along a set of lines to measure the number of line segments per unit length $n_{c}$ above a given height $z$ and the length distribution of these line segments $f(l)$. Equation (24) then allows us to evaluate the mean radius $\bar{a}$ of the contact spots in the corresponding three-dimensional section, and Eq. (25) determines the number of contact spots per unit area, $n$. Finally, Eq. (23) determines the probability density of the distribution for the contact radius.

This method gives good results for the distribution of contact spot sizes as long as the bearing area ratio is less than $10 \%$. For larger values of bearing area ratio, more complex contact spot geometries are obtained, ${ }^{9}$ some involving multiply connected areas-i.e., one or more regions of separation completely surrounded by contact. However, these conditions occur only under extremely high loads and are not of much practical interest.

\section{RESULTS}

As an example problem, we consider the contact between two half spaces over a square nominal contact area of size $1 \times 1 \mathrm{~mm}^{2}$ at various values of the bearing area ratio $B(z)$. The resistivity of both half spaces was taken to be $\rho_{1}=\rho_{2}=25 \times 10^{-9} \Omega \mathrm{m}$.

Figure 3 shows the variation of (a) the function $\bar{h}=n \bar{a}$ from Eq. (21) and (b) the electrical resistance $R_{e}$ with the 


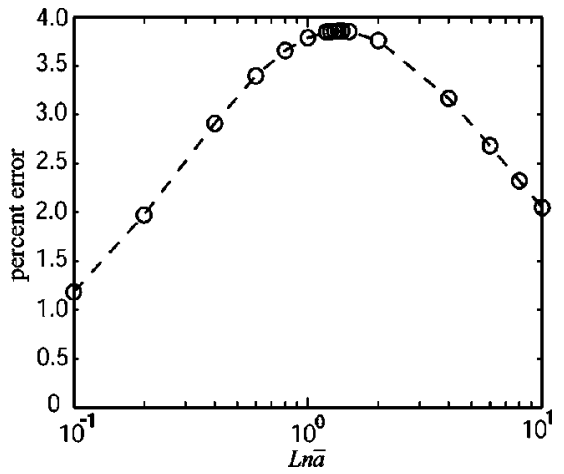

FIG. 4. Percentage difference between the predictions of the Greenwood equation (27) and the solution of Sec. III as a function of $L n \bar{a}$.

bearing area ratio $B(z)$. As we would expect, the contact resistance decreases with increasing bearing area ratio. For comparison, Nakamura ${ }^{7}$ showed that the electrical resistance for conduction through a single square contact spot of side $L$ is

$$
R_{e}^{S N}=\frac{0.868 \rho}{L} .
$$

Thus, if there were perfect electrical contact over the entire nominal contact area, the resistance would be $21.7 \mu \Omega$.

\section{A. Comparison with Greenwood's equation}

Equation (1) applies specifically to the case of a circular nominal contact area of radius $\alpha$, containing a distribution of $N$ contact areas, each of radius $a$. However, it is readily generalized to the present case by (i) replacing the cluster resistance term $\rho / 2 \alpha$ by $R_{e}^{S N}$ of Eq. (26) and (ii) replacing the product $N a$ by $L^{2} n \bar{a}$, giving

$$
R_{G}=\frac{\rho}{L}\left(\frac{1}{2 \operatorname{Ln} \bar{a}}+0.868\right) .
$$

This simple expression is shown by the dashed line in Fig. 3(b) and it clearly gives a very good approximation to the present numerical predictions. In fact, Eq. (27) is always slightly lower than the corresponding numerical calculation, the percentage difference being shown in Fig. 4 as a function of $n \bar{a}$. The reason for this difference is that the numerical treatment allows for the effect of the microscopic resistance in modifying the mean current density in the "cluster-scale" problem, whereas Eq. (27) assumes that the cluster resistance is always that which would be obtained in the perfect contact problem. This is most significant when the microscopic resistance is large, in which case the mean current density will be approximately uniform in the nominal contact area, rather than having the square-root singular behavior implied by $\mathrm{Na}-$ kamura's solution $^{7}$ and Eq. (26). However, in this limit, the resistance is dominated by the microscopic resistance term, and hence Eq. (27) still gives a good approximation to the numerical results. The maximum percentage difference therefore occurs at intermediate values of $\operatorname{Ln} \bar{a}$, being $3.86 \%$ at $\operatorname{Ln} \bar{a}=1.35$.

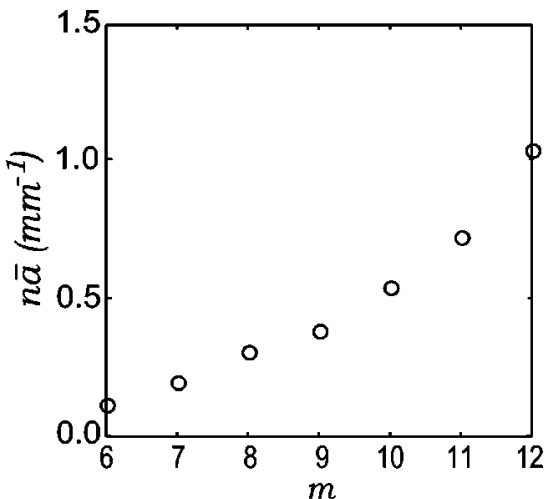

(a)

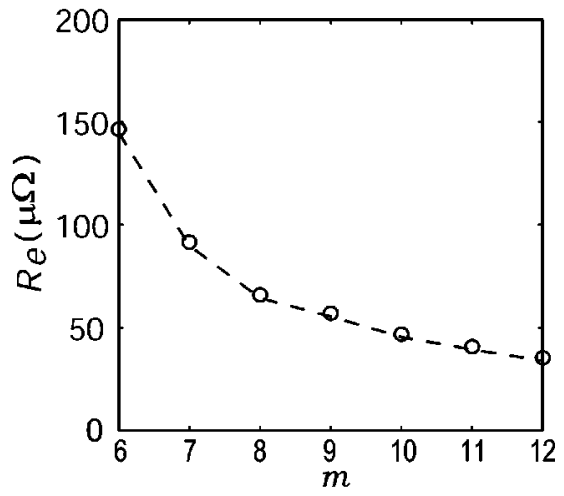

(b)

FIG. 5. Variation of (a) the function $\bar{h}=n \bar{a}$ from Eq. (21) and (b) the electrical contact resistance $R_{e}$ with sampling length $1 / 2^{m}$ for a bearing area ratio of 5\%. The dashed line in (b) was obtained from Eq. (27).

\section{B. Effect of sampling length}

Experimental measurements with the stylus profilometer show that, when using a large sampling interval, the surface exhibits only a few asperities with a large radius of curvature, whereas with a smaller sampling interval, larger numbers of asperities of smaller radius are revealed. Classical asperity-based models of contact appear to give reasonable predictions of electrical and thermal resistance, but it is not clear what sampling interval should be used in defining the resulting asperities. Ideally, we would hope that the predictions obtained using progressively refined surface descriptions would tend to a limit at small sampling length, thus providing some justification for truncating the description at a finite length scale.

This effect can be simulated in the present example by increasing the grid refinement of the RMD model. Figure 5(a) shows the function $\bar{h}=n \bar{a}$ from Eq. (21) as a function of grid refinement $m$ for a bearing area ratio of $5 \%$. This corresponds to the sampling of the rough surface at an interval of $1 / 2^{m} \mathrm{~mm}$. The results show a considerable increase in $\bar{h}$ with increasing $m$ and this translates to a comparable reduction of contact resistance $R_{e}$, as shown in Fig. 5(b). The dashed line in Fig. 5(b) corresponds to the Greenwood equation (27). These numerical calculations were extended to larger values of $n \bar{a}$ and confirm that the resistance tends to the perfect contact limit of Eq. (26) as $n \bar{a} \rightarrow \infty$. 


\section{DISCUSSION}

The multiscale model predicts a lower electrical contact resistance when a finer scale is used, since the finer scale reveals larger numbers of additional microscopic contact spots. In particular, the mean radius $\bar{a}$ decreases, but there is a larger increase in the number of contact spots per unit area $n$, leading to a net increase in $\bar{h}$. Similar behavior has been reported in other recent studies of the contact of quasifractal surfaces. $^{14,15}$

The "bearing area" hypothesis used in the example in Sec. V predicts a distribution that contains some relatively large contact spots along with increasingly large numbers of smaller spots as the sampling length is reduced. Similar characteristics are implied in the fractal contact model of Majumdar and Bhushan. ${ }^{12}$ This theory is most appropriate when the microscopic problem is dominated by plastic deformation, since in this case each local asperity contact is analogous to a hardness indentation. By contrast, the elastic contact theories of Borri-Brunetto et al. ${ }^{14}$ and of Ciaverella et al. ${ }^{15}$ predict that the size of all contact spots decreases with decreasing sampling length, so that in the theoretical fractal limit we have an infinite number of contact spots of zero size.

Elastic fractal contact theories also show that the product $\bar{h}=n \bar{a}$ would be unbounded in the limit $m \rightarrow \infty$, while the total area of actual contact

$$
A_{c}=\sum_{i=1}^{N} \pi a_{i}^{2}
$$

tends paradoxically to zero. ${ }^{14,15}$ If we define a new function $\psi(\xi, \eta)$ such that

$$
\psi(\xi, \eta) \equiv n \bar{a}[\phi(\xi, \eta)-\bar{\phi}(\xi, \eta)],
$$

and use this expression and Eq. (19) to substitute for $\bar{\phi}(\xi, \eta)$ and $\bar{h}(\xi, \eta)$ respectively in Eq. (9), we obtain

$$
\iint_{A} \frac{2 A h_{1}(\xi, \eta) \psi(\xi, \eta) d \xi d \eta}{\pi \sqrt{(x-\xi)^{2}+(y-\eta)^{2}}}+\frac{\psi(x, y)}{n \bar{a}}=\phi(x, y) .
$$

If $n \bar{a}$ increases without limit, the second term in Eq. (30) tends to zero, giving

$$
\iint_{A} \frac{2 A h_{1}(\xi, \eta) \psi(\xi, \eta) d \xi d \eta}{\pi \sqrt{(x-\xi)^{2}+(y-\eta)^{2}}}=\phi(x, y) .
$$

This equation has a bounded solution for $\psi(\xi, \eta)$ except along the boundary of the nominal contact area and hence in the fractal limit $\phi=\bar{\phi}$ from Eq. (29). In other words, the base potential becomes equal to the potential at the contact interface and Eqs. (16) and (12) give

$$
\frac{\rho}{2 \pi} \iint_{A} \frac{i(\xi, \eta) d \xi d \eta}{\sqrt{(x-\xi)^{2}+(y-\eta)^{2}}}=\phi(x, y),
$$

which is the equation defining perfect electrical contact throughout the nominal contact area $A$. Thus, any contact theory that predicts a distribution function $\bar{h}=n \bar{a}$ that increases without limit with decreasing sampling length will

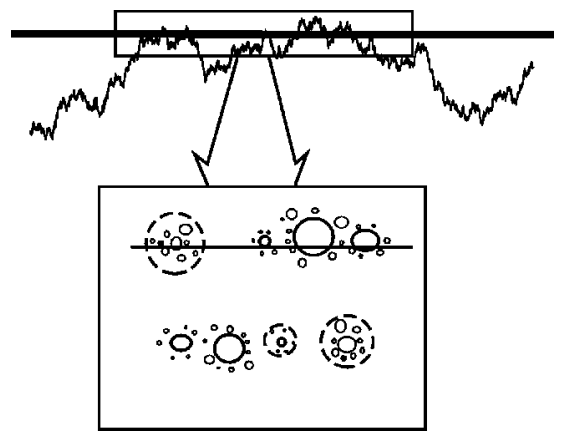

FIG. 6. Contact spot clusters due to large scale waviness of the surface. This tends to make the probability of a contact spot higher in the vicinity of other spots and lower in remote regions.

imply an electrical resistance equal to that based on the simple assumption of perfect electrical contact in the nominal contact area.

\section{A. Correlated distributions}

All of these results are predicted on the assumption in Sec. II that the distribution of contact spots is statistically uncorrelated-i.e., that the probability of a contact spot at $(x, y)$ is unaffected by the actual occurrence of a contact spot at a nearby point. Most theories of electrical and thermal contact resistance are based on this same assumption, since they draw on data about the height distributions of surface points, asperity summits, etc., but not on data about the relative spatial location of these features. However, it is in the nature of a multiscale surface that the distributions are correlated, since the larger scale waviness will tend to cluster the next scale of asperity contacts into groups, as shown in Fig. 6. Further evidence of the importance of correlation is provided by a recent result due to Barber, ${ }^{16}$ which places load-dependent bounds on the electrical contact resistance in an elastic contact problem, with the lower bound being generally tighter than the perfect contact limit.

Some allowance for the effect of clustering on the coarsest scale could be made by subdividing the nominal contact area and sampling the surface separately in each subarea generated. The results could then be used to define $h(x, y, a)$ as a piecewise constant function of $x, y$. The limitation of this technique is that the accuracy of the sampling technique of Sec. V depends upon the contact areas being substantially smaller than the subareas sampled.

A more promising approach to the inclusion of correlation effects might be to generalize the analysis of Sec. II to allow the function $h(x, y, a)$ to be correlated and to devise a way to estimate the correlation functions for the distribution from measurements of the correlation of the line segment distribution $f(l)$ of Eq. (23). These questions are the subject of ongoing research.

\section{CONCLUSIONS}

We have presented a model for the electrical contact of rough surfaces, extending Greenwood's equation for conduction through a cluster of circular contacts to a system in which the probability of a contact spot at a given location is defined in statistical terms. The model was illustrated using a 
mathematically generated surface with fractal characteristics and the bearing area hypothesis, properties of the surface being determined using a relation between three-dimensional properties and profile properties due to Jang. ${ }^{9}$ We show that in the fractal limit the theory would predict effectively perfect electrical contact throughout the nominal contact area, suggesting that the correlation between the location of adjacent contact spots needs to be taken into account.

\section{ACKNOWLEDGMENT}

Y. H. J. is pleased to acknowledge support from the Basic Research Program of the Korea Science and Engineering Foundation under Grant No. R08-2003-000-10162-0.

${ }^{1}$ J. A. Greenwood, Br. J. Appl. Phys. 17, 1621 (1966).

${ }^{2}$ R. Holm, Electrical Contacts, Theory and Applications (Springer-Verlag, Berlin, 1967).

${ }^{3}$ L. Boyer, S. Noel, and F. Houze, IEEE Trans. Compon., Hybrids, Manuf. Technol. 14, 134 (1991).
${ }^{4}$ R. D. Malucci, in Proceedings of the 36th IEEE Holm Conference on Electrical Contacts, 1990, p. 625.

${ }^{5}$ R. D. Malucci, IEEE Trans. Compon., Hybrids, Manuf. Technol. 15, 339 (1992).

${ }^{6}$ M. D. Bryant, IEEE Trans. Compon., Hybrids, Manuf. Technol. 17, 89 (1994).

${ }^{7}$ M. Nakamura, IEEE Trans. Compon., Packag. Manuf. Technol., Part A 18, 382 (1994).

${ }^{8}$ L. Boyer, IEEE Trans. Compon., Hybrids, Manuf. Technol. 24, 50 (2001).

${ }^{9} \mathrm{Y}$. H. Jang (in press).

${ }^{10}$ J. A. Greenwood and J. B. P. Williamson, Proc. R. Soc. London, Ser. A 295, 300 (1966).

${ }^{11}$ J. A. Greenwood and J. H. Tripp, J. Appl. Mech. 34, 153 (1967).

${ }^{12}$ A. Majumdar and B. Bhushan, J. Tribol. 113, 1 (1991).

${ }^{13}$ R. F. Voss, in Fundamental Algorithms for Computer Graphics, edited by R. A. Earnshaw, NATO ASI Series F17 (Springer-Verlag, Berlin, 1985), pp. $805-835$.

${ }^{14}$ M. Borri-Brunetto, A. Carpinteri, and B. Chiaia, Probamat-21st Century: Probabilities and Materials, edited by G. Frantziskonis (Kluwer Academic Publishers, Dordrecht, 1998), pp. 45-64.

${ }^{15}$ M. Ciaverella, G. Demelio, J. R. Barber, and Y. H. Jang, Proc. R. Soc. London, Ser. A 456, 387 (2000).

${ }^{16}$ J. R. Barber, Proc. R. Soc. London, Ser. A 459, 53 (2003). 